\title{
LA ENSEÑANZA UNIVERSITARIA ESPAÑOLA EN MATERIA DE EDUCACIÓN MEDIÁTICA
}

\author{
Spanish undergraduate teaching in media education field
}

\begin{abstract}
Auotres: MASANET, Maria-Jose y FERRÉS, Joan
Becaria Formación Personal Investigador (FPI) y miembro del grupo UNICA del Departamento de Comunicación de la Facultad de Comunicación de la Universidad Pompeu Fabra. Roc Boronat, 138, 08018, Barcelona.Tel.: +34935422344 - mjose.masanet@upf.edu

Profesor Titular, coordinador docente de los estudios de Comunicación Audiovisual y miembro del grupo UNICA del Departamento de Comunicación de la Facultad de Comunicación de la Universidad Pompeu Fabra de Barcelona. Roc Boronat, 138, 08018, Barcelona. Tel.: +34935422344-joan.ferres@upf.edu
\end{abstract}

\section{Resumen}

En las últimas décadas han proliferado notablemente los estudios alrededor de la Educación Mediática en el ámbito nacional e internacional y se ha corroborado la falta de competencia mediática por parte de la ciudadanía y la necesidad de ampliar las investigaciones en esta línea para encontrar causas y soluciones a la situación actual. En este contexto, el artículo pretende incidir en el estudio del ámbito de la enseñanza universitaria. Se parte de la convicción de que, para optimizar la competencia mediática de la ciudadanía, habría que incidir en los profesionales de la comunicación, responsables de los mensajes, y en los profesionales de la educación, que tienen incidencia sobre los interlocutores de éstos. Y el círculo se cierra en la universidad. Por lo tanto, el artículo tiene el objetivo de detectar en los planes de estudio del ámbito universitario necesidades y carencias que provoquen déficit en la competencia mediática de la ciudadanía. Para ello, se ha administrado un cuestionario a los responsables de todas las titulaciones universitarias relacionadas con la comunicación y con la educación de España y, a través del análisis de contenido, se han sometido a análisis sus planes de estudio y guías docentes. Los resultados son reveladores: hay carencia de oferta docente de asignaturas directamente relacionadas con la educación mediática. Y la mayor parte de las asignaturas indirectamente relacionadas se centran en la dimensión tecnológica. Por otro lado, la mayor parte de las asignaturas que los responsables académicos consideran directamente relacionadas son indirectas, según el análisis de contenido.

\section{Palabras clave}

Educación Mediática, Plan de estudios, Universidad, Comunicación, Educación.

\begin{abstract}
In recent decades, studies about Media Education have proliferated in both national and international levels. It is widely accepted the citizens' lack of Media Literacy and their need to expand the research by focusing on its causes and solutions. In this context, this article aims to examine the undergraduate teaching and the way universities deal with the problem of media literacy. We understand that in order to optimize the general media competence, we should influence on communication professionals, who are responsible for the media messages; and on education professionals, who have an impact on the active audiences. All these actors confluence in a single space: the university. Therefore, the work aims to identify the needs and lacks in the curriculum of the media and education studies which eventually lead to Media Literacy deficit of citizens. For this purpose, a questionnaire was administered to the heads of all Spanish undergraduate studies related to communication and education. Additionally, their curricula and syllabus were analyzed through content analysis. The results are revealing. First, there is a lack of courses directly related to Media Education. Second, most of the courses indirectly related to it are focused on the technological dimension. Finally, based on the content analysis, most of the courses considered by the professors directly related to media education have in fact an indirect connection.
\end{abstract}

\section{Key words}

Media Education, Curriculum, University, Communication, Education. 


\section{Introducción}

Los estudios en torno a la Educación Mediática han proliferado notablemente en los últimos años, dada la importancia y presencia de los medios de comunicación en la sociedad actual. En el ámbito europeo las aportaciones han propiciado un nuevo panorama académico en el que la Educación Mediática ocupa un lugar destacado. La Declaración de Praga (2003), la Proclamación de Alejandría (2005), la Agenda de París (2007), la Declaración de Lima (2009), la Declaración de Paramillo (2010), la Declaración de Braga (2011), la Declaración de Maceió (2011) la Declaración de Fez (2011) o la Declaración de La Habana (2012) son algunos ejemplos de la importancia que la educación mediática ha cobrado en el contexto actual e internacional.

En el entorno español también se han llevado a cabo iniciativas interesantes, aunque de momento han sido casos puntuales. En este contexto, destaca la investigación dirigida por Joan Ferrés et al. (2011), centrada en el análisis del grado de competencia mediática de la ciudadanía en España. En la investigación participaron universidades de las 17 comunidades autónomas y fue financiada por el CAC (Consejo del Audiovisual de Cataluña), el Ministerio de Educación y entidades pertenecientes a las diferentes comunidades autónomas. La primera fase de la investigación se centró en la definición de las dimensiones e indicadores que conforman la Competencia Mediática (Ferrés, 2006; 2007). Un total de 46 expertos del ámbito iberoamericano y 14 del español participaron en la elaboración del documento, que sirvió de base para las siguientes etapas de la investigación. En la segunda fase, se administraron 6.626 cuestionarios, se realizaron 31 entrevistas en profundidad y 28 grupos de discusión.

Los resultados pusieron de manifiesto una situación preocupante: en cinco de las seis dimensiones que componen la competencia mediática no se llegó al aprobado, siendo la dimensión tecnológica la única excepción. En base a los resultados, las conclusiones de los autores fueron claras:

Las graves carencias en contenidos relativos a los distintos ámbitos de la competencia mediática deberían llevar a los responsables del mundo educativo a potenciar la educación mediática en todos los niveles tanto de la enseñanza formal como de la no formal (Ferrés, Aguaded-Gómez y GarcíaMatilla, 2012: 40).

Teniendo en cuenta los resultados obtenidos y la preocupación de los investigadores por el desarrollo de este campo de estudio, esta línea de investigación ha continuado activa y, en el año 2012, en base a los cambios tecnológicos, Ferrés y Piscitelli (2012) actualizaron el documento de dimensiones e indicadores que componen la Educación Mediática. Esta actualización se realizó a partir de las propuestas de más de 50 expertos españoles y extranjeros. En palabras de los autores:

Desde el año 2005 hasta hoy han cambiado mucho las cosas en el ámbito de la comunicación mediática. Se ha producido una importante transformación del paisaje comunicativo, provocada por la aparición de nuevos dispositivos tecnológicos y de nuevas prácticas comunicativas. Estos cambios obligan a introducir modificaciones en la definición de la competencia mediática, ajustando la formulación de las dimensiones e incorporando nuevos indicadores (Ferrés y Piscitelli, 2012: 76).

Actualmente, la investigación se amplía y complementa a través de un proyecto $\mathrm{I}+\mathrm{D}+\mathrm{i}$, financiado por el Ministerio de Economía y Competitividad. Se trata de un proyecto coordinado entre la Universidad de Huelva, con Ignacio Aguaded como Investigador Principal, la Universidad de Segovia, con Agustín García Matilla como Investigador Principal y la Universidad Pompeu Fabra, con Joan Ferrés como Investigador Principal y coordinador de los tres subproyectos.

Mediante esta iniciativa se pretende detectar necesidades y carencias en la competencia mediática, a través del análisis de tres sectores sociales clave: los profesionales de la comunicación, los profesionales de la enseñanza obligatoria y los de la enseñanza universitaria, tanto en el ámbito de la comunicación (Comunicación Audiovisual, Periodismo y Publicidad y Relaciones Públicas) como en el de la educación (Magisterio, Pedagogía y Educación Social).

En este contexto, el artículo se centra en los resultados obtenidos en el subproyecto dirigido por Joan Ferrés y titulado La enseñanza universitaria ante la 
competencia mediática en un entorno digital, que se centra en el análisis de la enseñanza universitaria española en relación a la oferta docente en materia de educación mediática.

\section{Objetivos}

El objetivo del proyecto es detectar en los planes de estudio del ámbito universitario necesidades y carencias que provoquen déficit en la competencia mediática de la ciudadanía. Para ello, se han seleccionado todas las titulaciones relacionadas con la comunicación y con la educación de todas las universidades de España y se han sometido a análisis sus planes de estudio y sus guías docentes. Para justificar esta elección hay que advertir que se parte de la convicción de que toda experiencia de comunicación audiovisual es el resultado de la interacción entre unos mensajes y unos interlocutores de los mismos. Para optimizar la competencia mediática de la ciudadanía habría que incidir, pues, en los profesionales de la comunicación, responsables de los mensajes, y en los profesionales de la educación, que tienen una incidencia directa sobre los interlocutores de estos mensajes. Y este círculo se cierra en la universidad, donde están los formadores tanto de los profesionales de la educación como de los de la comunicación.
En el artículo se presentan los resultados preliminares fruto de una primera fase de análisis de los planes de estudio y de las guías docentes, que aporta datos sobre la presencia de asignaturas relacionadas con la Educación Mediática en el ámbito español y hace una catalogación de éstas entre directas e indirectas. También se aportan datos sobre la concepción que los responsables académicos de las universidades de España tienen sobre la oferta docente de Educación Mediática.

En la segunda fase, una vez catalogadas las guías como directas o indirectas, se procederá al análisis de contenido en profundidad. Las guías serán analizadas en base a todos los ítems relativos a las dimensiones e indicadores que componen la competencia mediática, siguiendo la actualización de Ferrés y Piscitelli (2012). Esta detallada catalogación nos aportará información referente a la atención que las guías docentes de Educación Mediática prestan a cada una de las dimensiones y, por lo tanto, permitirá descubrir cuáles son las dimensiones e indicadores más atendidos y cuáles los más olvidados.

\section{Metodología}

Para llevar a cabo la investigación, se ha utilizado una metodología que combina el análisis cuantitativo, a través de la encuesta sociológica, y el cualitativo, a través del análisis de contenido. Por lo tanto, se han seleccionado dos muestras de estudio.

Para el análisis cuantitativo, se recurre a una base de datos que se creó en el año 2011, a partir de la información extraída de la página oficial del Ministerio de Educación, Cultura y Deporte, en el apartado de oferta de titulaciones ${ }^{1}$, centrándose en todos los grados de educación y comunicación que se ofrecían en España. La base de datos incluye 393 estudios de grado en comunicación y en educación en

${ }^{1}$ https://www.educacion.gob.es/notasdecorte/jsp/compBdDo.do $(08 / 03 / 2013)$ el conjunto de las universidades españolas y en ella se cuenta con información sobre la universidad y departamento que ofrece cada uno de los estudios, el nombre del decano o director y los contactos pertinentes. Los investigadores hicieron llegar el cuestionario, vía correo electrónico, a los responsables de estos 393 estudios. Finalmente, la muestra de estudio quedó constituida por los 354 estudios de los que se consiguieron las informaciones requeridas.

Para el cuestionario se utilizó la herramienta informática Google Docs. El cuestionario incluía preguntas identificativas, de selección múltiple, dicotómicas, y preguntas abiertas. El objetivo del mismo era extraer información sobre la presencia o ausencia de asignaturas relacionadas con la educación mediática en los estudios, las características de éstas 
(créditos, carácter, formación profesores, etc.) y la valoración que la educación mediática recibía por parte de los encargados académicos, jefes de estudio o similares. Se llevó a cabo la prueba piloto con estudios de distintas universidades, se modificaron algunas preguntas y se procedió al pase definitivo. Se envió el cuestionario, junto con una carta explicativa sobre el proyecto, por correo electrónico, a los contactos de la base de datos de cada estudio. En el correo se les pedía que rellenaran el cuestionario, previa consulta con el jefe de estudios, responsable académico, coordinador del Grado, coordinador del Equipo Docente, responsable de Innovación Docente de su facultad o similar. Cuando no se recibía respuesta por correo electrónico, los investigadores rellenaban una parte del cuestionario con la información de la web de la universidad y hacían las llamadas pertinentes para rellenar las otras cuestiones. El trabajo de campo se desarrolló durante el año 2012 y los datos fueron introducidos en el programa estadístico SPSS. Se llevó a cabo un análisis estadístico univariable y bivariable, adoptando el nivel de significación de valor 0,05 . Se utilizaron recursos comunes de la estadística: tablas de frecuencia y tablas de contingencia.

La muestra de estudio para el análisis de contenido se determinó a partir de las respuestas a los cuestionarios. En primer lugar, se extrajo la información sobre todas las asignaturas a las que se hizo referencia en los cuestionarios (461). En segundo lugar, se revisaron los planes de estudio de todos los estudios que componían la base de datos inicial, para determinar qué asignaturas de las citadas en el cuestionario se centraban en los distintos aspectos de la educación mediática y, por último, se descargaron las guías docentes de las asignaturas que cumplieron los mínimos establecidos para ser consideradas de Educación Mediática. Se obtuvieron 325 guías docentes, que constituyen la muestra para el análisis de contenido.

Para esta fase de la investigación se creó una tabla de análisis basada en las dimensiones e indicadores que componen la competencia mediática (Ferrés y Piscitelli, 2012). La tabla era una reestructuración del documento base sobre dimensiones e indicadores, que permitía catalogar las guías en directa o indirectamente relacionadas con la Educación Mediática, en función de la mayor o menor atención que prestaban a cada una de las dimensiones e indicadores. El objetivo era determinar qué guías tratan la Educación Mediática en su globalidad y cuáles se centran, únicamente, en alguna dimensión, como la tecnológica. En base a lo expuesto, se estableció que se considerarían asignaturas directamente relacionadas con la Educación Mediática aquellas que en su guía docente trataban con una cierta profundidad al menos 4 de las 6 dimensiones. En una primera revisión se observó que algunas guías llegaban a mencionar 4 y hasta 5 dimensiones, pero lo hacían de manera muy superficial. En estos casos, eran consideradas indirectamente relacionadas. Mediante este análisis se obtuvieron datos para hacer un análisis comparativo con los datos obtenidos de la administración de los cuestionarios.

\section{Resultados}

Los resultados de los cuestionarios distribuyen la muestra por estudios de manera desigual. Se obtuvieron 87 respuestas de estudios de Educación Infantil (24,6\%), 81 de Educación Primaria (22,9\%), 49 de Comunicación Audiovisual (13,8\%), 41 de Periodismo (11,6\%), 37 de Educación Social (10,5\%), 36 de Publicidad y Relaciones Públicas $(10,2 \%)$ y 23 de Pedagogía $(6,5 \%)$. Un total de 354 estudios, $228(64,4 \%)$ del ámbito de la educación y $126(35,6 \%)$ del de la comunicación. Es una proporción lógica, dado que los estudios de educación están mucho más extendidos por todo el territorio español.
En lo referente a la presencia o ausencia de asignaturas directamente relacionadas con la Educación Mediática, el 58,2\% (N=206) afirmó que había alguna asignatura en el plan de los estudios y el $41,8 \%(N=148)$ que no. El porcentaje de respuestas afirmativas disminuyó cuando se les preguntó si tenían una segunda asignatura relacionada directamente con la Educación Mediática: solo el 19,8\% $(\mathrm{N}=70)$ afirmó tenerla, frente al 80,2\% ( $\mathrm{N}=284)$ que negó que la hubiera en su plan de estudios. Y el porcentaje continúo disminuyendo en la pregunta sobre si había una tercera asignatura relacionada directamente, solo el 7,6\% (N=27) respondió sí y el 92,4\% $(\mathrm{N}=327)$ 
no. Cada vez que respondían afirmativamente a la presencia de asignaturas directamente relacionadas con la Educación Mediática, se les pedía que

ofrecieran información sobre éstas (nombre, créditos, carácter, profesores, etc.). Si se suman las cifras de todas las asignaturas directamente relacionadas que se mencionaron en el cuestionario (206, 70 y 27), se obtiene un total de 303 asignaturas en todo el ámbito universitario español.

Similar fue el caso de las asignaturas indirectamente relacionadas con la Educación Mediática. El porcentaje fue disminuyendo a medida que aumentaba el número de asignaturas. En la primera pregunta, sobre si estaba prevista en el plan de estudios alguna asignatura indirectamente relacionada con la Educación Mediática, se obtuvieron 124 (35,0\%) respuestas afirmativas y 230 negativas $(65 \%)$. En este caso, se preguntó también si se ofrecían actualmente en el plan de estudios, ya que hay asignaturas que constan en los planes de estudio pero no se ofrecen a los estudiantes. El número de asignaturas disminuyó ligeramente: el 97,6\% ( $\mathrm{N}=121)$ se ofrecían y el 2,4\% $(\mathrm{N}=3)$ no. A la pregunta sobre si había una segunda asignatura relacionada indirectamente con la Educación Mediática, solo el 7,3\% $(\mathrm{N}=26)$ respondió de forma afirmativa y el 92,7\% ( $=328)$ negativa. Por último, cuando se les preguntó si había una tercera asignatura relacionada directamente, se obtuvieron $11(3,1 \%)$ respuestas afirmativas y 343 $(96,9 \%)$ negativas. Sumando las cifras de todas las respuestas afirmativas $(121,26$ y 11$)$ se obtienen un total de 158 asignaturas.

Si se relacionan los datos sobre el estudio con la presencia o no de una asignatura directamente relacionada (Tabla. 1), se observa que la relación es significativa, obteniendo un valor $\mathrm{p}=0,033$. Es significativo el hecho de que, en el ámbito de los estudios de comunicación, en casi todos los casos, exceptuando el de periodismo, han respondido más encuestados "no" que "si" a la pregunta, contrariamente a lo que pasa en los estudios de educación, donde hay muchas más respuestas afirmativas: E. Infantil $(66,7 \% ; N=58)$, E. Primaria
$(64,2 \% ; \quad N=52), \quad$ E. Social $(62,2 \% ; \quad N=23)$ y Pedagogía $(65,2 \% ; N=15)$.

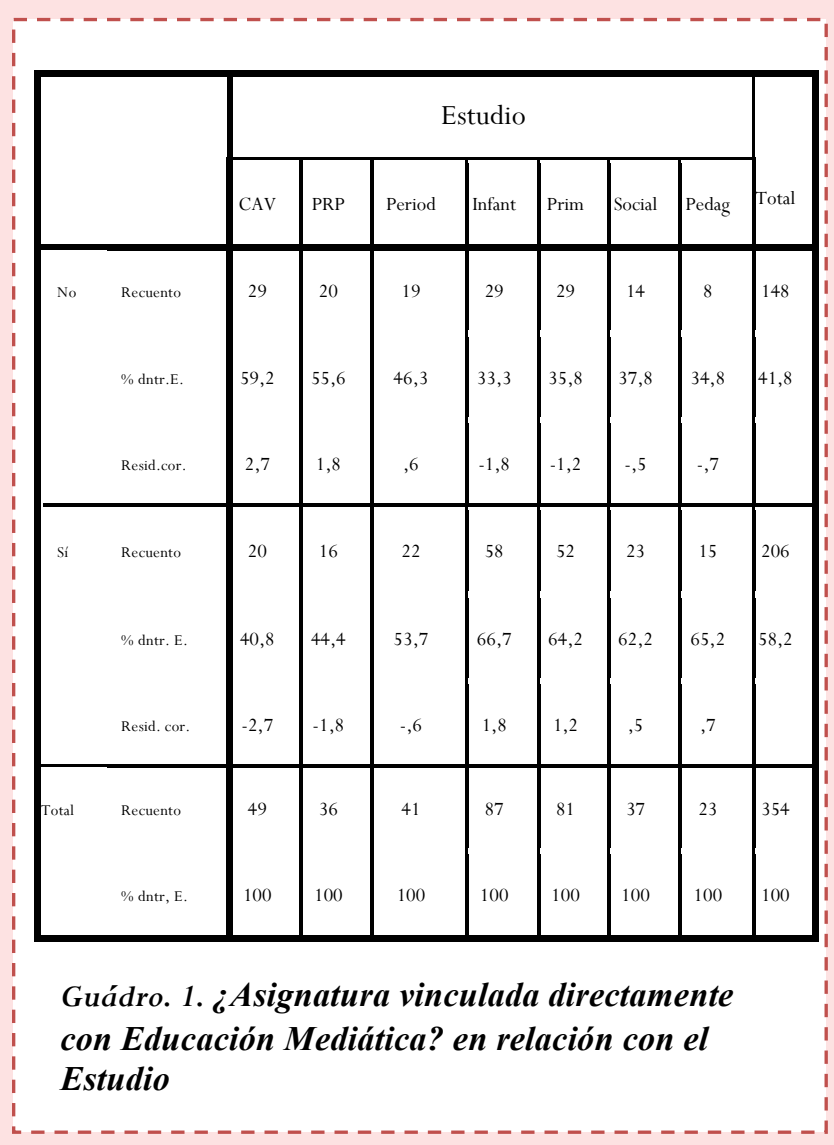

En el caso de la relación entre el estudio y los datos de la pregunta sobre si está prevista alguna asignatura indirectamente relacionada con la Educación Mediática (Tabla. 2), de nuevo se obtiene una relación significativa, con un valor $p=0,007$. Cabe destacar que, en este caso, las respuestas negativas de los estudios de educación son muy superiores a las afirmativas. En E. Infantil $(73,6 \% ; \mathrm{N}=64), \quad \mathrm{E}$. Primaria $(75,7 \% ; N=28)$ y E. Social $(75,7 \% ; N=28)$ se sobrepasa el $70 \%$ de respuestas negativas. El caso de Pedagogía $(69,6 \% ; \mathrm{N}=16)$ no supone ninguna excepción, ya que el porcentaje es igualmente alto. Por el contrario, en los estudios de comunicación las cifras de las respuestas afirmativas y negativas se ajustan al 50\%. 
Guádro. 2. ¿Está prevista en el plan de estudios alguna asignatura relacionada con la Educación Mediática? en relación con el Estudio

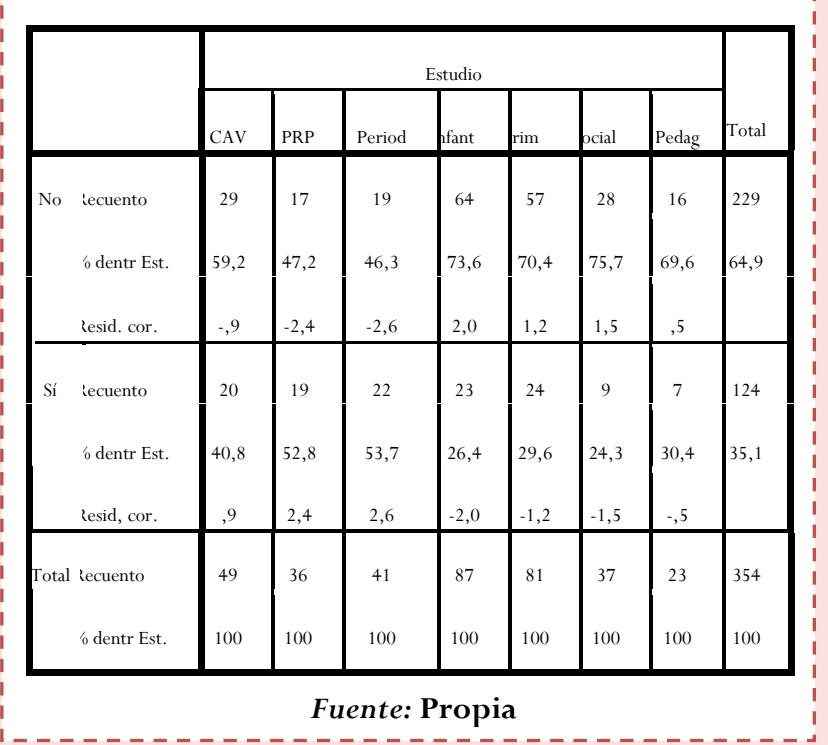

Atendiendo a los datos obtenidos en el análisis de contenido, los resultados son contradictorios. A partir del análisis de los planes de estudio, siguiendo la tabla de catalogación de creación propia, de cada uno de los estudios de los que se obtuvo respuesta en el cuestionario se desprende que los responsables académicos, coordinadores de grado, coordinadores de equipo, etc., no distinguen entre las asignaturas que están directamente relacionadas con la Educación Mediática y las que lo están indirectamente. Los resultados son reveladores: $72(22,15 \%)$ asignaturas relacionadas directamente con la Educación Mediática y $253(77,85 \%)$ indirectamente. Esto hace un total de 325 asignaturas, en contraposición a las $461-303$ directas $(65,73 \%)$ y 158 indirectas $(34,27 \%)$ - que se citaron en el cuestionario. En los estudios del ámbito de la comunicación se catalogaron $17(15,74 \%)$ asignaturas como directamente relacionadas y 91 $(84,26 \%)$ como indirectamente. Similar es el caso de las asignaturas del ámbito de la educación, 56 $(26,17 \%)$ relacionadas directamente y $158(73,83 \%)$ indirectamente. Como se puede observar, el análisis de contenido de los planes de estudio y de las asignaturas relacionadas directa o indirectamente con la educación mediática desvela que la presencia de asignaturas indirectamente relacionadas con la educación mediática es mucho mayor que la de directas, tanto en los estudios de comunicación como en el de los de educación, contrariamente a lo se respondió en el cuestionario.

Asimismo, cabe destacar que, aparte de las asignaturas que no guardan ninguna relación con la Educación Mediática y, por lo tanto, no entraron en los recuentos, hay una tendencia generalizada a considerar como asignaturas directamente relacionadas con la Educación Mediática algunas que se centran exclusivamente en la dimensión tecnológica (como las de las TICs aplicadas a la educación) o en la deontología o ética profesional (en los estudios de Comunicación).

Gráfico no1: Total asignaturas relacionadas directamente e indirectamente con la EM. Datos extraidos del cuestionario.

Directamente relacionadas

- Indirectamente Relacionadas

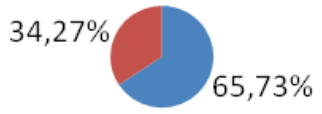

Gráfico n-2: Total asignaturas relacionadas directamente e indirectamente con la EM. Datos extraidos del análisis de contenidos.

Directamente relacionadas

- Indirectamente Relacionadas

$22,15 \%$

$77,85 \%$

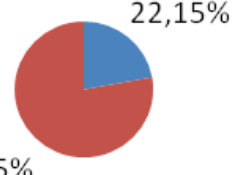

Fuente: Propia

Volviendo a los resultados del cuestionario, para obtener información complementaria sobre la oferta docente en educación mediática se les hicieron diversas preguntas. En referencia a la existencia de seminarios, jornadas o actividades extraacadémicas en los grados vinculados a la temática, la respuestas son más complejas: un 57,6\% ( $\mathrm{N}=204)$ no sabe de la existencia de estos o no contesta por la falta de información al respecto. Solo el 25,4\% (N=90) afirma que existen y el $16,9 \% \quad(N=60)$ que no existen. Cuando se les pregunta a los que han 
respondido afirmativamente si estos cursos se interrelacionan con los ámbitos educativos y sus profesionales, el $92,1 \% \quad(\mathrm{~N}=82) \quad$ responde afirmativamente y el 7,9\% negativamente $(\mathrm{N}=7)$. Similar es el caso de la presunta sobre la existencia de asignaturas en máster o postgrado relacionadas directamente con la Educación Mediática: el 42,1\% $(\mathrm{N}=149)$ responden que no saben de la existencia o no contestan por falta de información. El 33,9\% $(\mathrm{N}=120)$ confirma la existencia y el $24 \%(\mathrm{~N}=85)$ la no existencia. Cuando se les pregunta si se plantean actividades docentes que conecten con experiencias de innovación pedagógica sobre Educación Mediática, el 40,4\% ( $\mathrm{N}=143)$ no responde, el 42,7\% ( $\mathrm{N}=151)$ responde que sí y el 16,9\% (N=60) que no.
Por último, cuando se pregunta a los encuestados si consideran que la oferta docente de sus estudios en materia de Educación Mediática es suficiente, dejando fuera los casos perdidos, que son los que no respondieron a la pregunta, el $74,1 \% \quad(\mathrm{~N}=237)$ responden afirmativamente y el 25,9\% (N=83) negativamente. Por lo tanto, se observa que un gran porcentaje de los encuestados considera que la oferta docente en Educación Mediática de los distintos estudios es la correcta. Es un dato curioso, dado que la mayor parte de los encuestados no distingue entre asignaturas relacionadas directa o indirectamente, teniendo en cuenta la contraposición de resultados entre el cuestionario y el análisis de contenido.

\section{Conclusiones}

Los resultados de la investigación previa (Ferrés et al., 2011) ya nos alertaron de la necesidad de una Educación Mediática para el conjunto de la sociedad y en la primera fase de la investigación actual se confirma que una de las causas de los malos resultados en competencia mediática es la falta de oferta docente en educación mediática en las universidades de educación y de comunicación, lo que provoca unos profesionales de la educación y de la comunicación con graves carencias en el tema. Así mismo, uno de los principales motivos de esta carencia es la falta de conocimiento o compromiso de los responsables académicos hacia la Educación Mediática, ya que, como desvelan los resultados, no son capaces de distinguir entre asignaturas directa o indirectamente relacionadas con la Educación Mediática. Éste hecho provoca que haya muchas asignaturas indirectamente relacionadas, la mayoría únicamente centradas en las TIC, que son consideradas directamente relacionadas y, por lo tanto, con éstas, se dé por supuesto que ya existe una oferta docente que cubre la Educación Mediática, cuando lo que cubre es una de las dimensiones de esta competencia.

\section{Apoyos}

Esta investigación forma parte del Proyecto I+D del Ministerio de Economía y Competitividad con clave: EDU2010-21395-C03, titulado "La competencia en comunicación audiovisual en un entorno digital. Diagnóstico de necesidades en tres ámbitos sociales".

\section{Referencias}

- ANABISAI y UNET (2010): “Manifestación de Paramillo", en https://docs.google.com/file/d/0B8nzryP1iqFlMGQ 3N2I5YmYtNDYwNi00MjdjLWFmMjEtOWNiZmU2 ZGQ0OGMz/edit?hl=en US (consultado: 16/04/2013)

- Comisión Nacional de la UNESCO et al. (2011): "Declaração de Braga sobre alfabetización mediática", en $\underline{\text { http://www.cca.eca.usp.br/noticia/756 }}$ (consultado: 10/04/2013)

- Comisión Nacional de los EEUU De Norteamérica para las Bibliotecas y la Documentación et al. (2003): "Declaración de Praga. Hacia una sociedad alfabetizada en información”, en http://sole.com/plec/archivos/Docs_Bibliografias/Declaraci_P raga castellano.pdf (consultado: 16/04/2013) 
- Declaración de la Habana (2012): “15 acciones de ALFIN por un trabajo colaborativo y de generación de redes para el crecimiento de la alfabetización informacional en el contexto de los países iberoamericanos", en http: / / www.alfared.org/sites/www.alfared.org/files /Declaraci\%C3\%B3n\%20de\%20La\%20Habana.\%20A LFIN\%20desde\%20Iberoam\%C3\%A9rica\%20\%28201 2\%29.pdf (consultado: 10/04/2013)

- Expertos reunidos en el I Foro Internacional sobre Alfabetización Informacional y Mediática (2011): "Fez Declaration on media and information Literacy", en http://www.unesco.org/new/fileadmin/MULTIME DIA/HQ/CI/CI/pdf/news/Fez\%20Declaration.pdf (consultado: 16/04/2013)

- Expertos reunidos en el XXIV Congreso Brasilero de Biblioteconomía (2011): "Declaraçao de Maceió sobre a competencia em informaçao", en http: / / www.alfared.org/sites/www.alfared.org/files /DECLARA $\% \mathrm{C} 3 \% 87 \% \mathrm{C} 3 \% 83 \mathrm{O} \% 20 \mathrm{DE} \% 20 \mathrm{MACEI}$ \%C3\%93\%20SOBRE\%20AS\%20COMPET\%C3\%8A NCIAS\%20EM\%20INFORMA\%C3\%87\%C3\%83O.p df (consultado: 16/04/2013)

- Ferrés, Joan, Aguaded-Gómez, Ignacio y GarcíaMatilla, Agustín (2012): "La competencia mediática de la ciudadanía española: dificultades y retos", en Icono 14, vol. X, no 3, pp. 23-42

- Ferrés, Joan y Piscitelli, Alejandro (2012): “La competencia mediática: propuesta articulada de dimensiones e indicadores", en Comunicar, vol.XIX, $\mathrm{n}^{\circ}$ 38, pp. 75-82
- Ferrés, Joan et al. (2011): Competencia mediática. Investigación sobre el grado de competencia de la ciudadanía en España. España, Ministerio de Educación (Instituto de Tecnología Educativa), Consell de l' Audiovisual de Catalunya y Grupo Comunicar.

- Ferrés, Joan (2007): "La competencia en comunicación audiovisual: dimensiones e indicadores", en Comunicar, vol. XV, no 29, pp. 100-107.

- Ferrés, Joan (2006): "La competencia en comunicación audiovisual: propuesta articulada de dimensiones e indicadores”, en Quaderns del CAC, n 25, pp. 9-17

- UNESCO (2005): "The Alexandria Proclamation on Information Literacy and Lifelong Learning", en http: / / archive.ifla.org/III/wsis/BeaconInfSoc.html (consultado: 10/04/2013)

- UNESCO (2007): "Paris Agenda or 12 Recommendations for Media Education", en http://www.diplomatie.gouv.fr/fr/IMG/pdf/Parisag endafin en.pdf (consultado: 10/04/2013)

- UNESCO (2009): "Declaración de Lima. Alfabetización Informacional: Formando a los Formadores", en http: / / taller.pucp.edu.pe/alfabetizacion/ (consultado:

$16 / 04 / 2013)$

\section{Forma de Citación}

MASANET, Maria-Jose y FERRÉS, Joan: La enseñanza universitaria española en materia de educación mediática. Revista Communication Papers, $\mathrm{N}^{\circ}$ 2, Monográfico 1, páginas 83 a 90. Departamento de Filología y Comunicación de la Universidad de Girona. Recuperado el _ de de 2 de: http://www.communicationpapers.es 